\title{
Claudin-18 expression under hypoxia in neonatel lungs of brochopulmonary dysplasia model rats
}

\author{
Jingye Zuo ${ }^{1}$, Yajie Tong ${ }^{1}$, Yuting Yang ${ }^{1}$, Yirui Wang ${ }^{1}$, and Dongmei Yue ${ }^{1}$ \\ ${ }^{1}$ Shengjing Hospital of China Medical University
}

December 24, 2021

\begin{abstract}
Background: Bronchopulmonary dysplasia (BPD) is characterized by impaired alveolar and microvascular development. Claudin18 is the only known lung-specific tight junction protein affecting alveolar epithelium development and transdifferentiation. Objective: To explore the changes in claudin-18 expression, alveolar epithelial cell (AEC) marker proteins, the canonical Wnt pathway, and their possible regulatory relationships in a hyperoxia-induced BPD rat model. Methods: The BPD neonatal rat model was established by exposure to hyperoxia (85\%). Hematoxylin and eosin (HE) staining was used to confirm the establishment of the BPD model. The mRNA levels were assessed using quantitative real-time polymerase chain reaction, while protein expression levels were determined using western blotting, immunohistochemical staining, and immunofluorescence . Results: As confirmed by HE staining, the BPD neonatal rat model was successfully established. Compared with the air group, claudin-18 and claudin- 4 expression decreased in the hyperoxia group. The expression of $\beta$-catenin of the Wnt signaling decreased, whereas that of p-GSK-3 $\beta$ increased. Expression of the AEC II marker SFTPC decreased initially and then increased, whereas that of the AEC I marker Podoplanin increased on day $14(\mathrm{P}<0.05)$. Conclusions: Claudin-18 downregulation during hyperoxia may affect lung development and maturation, which may result in hyperoxia-induced BPD. Additionally, claudin-18 is associated with the canonical Wnt pathway and alveolar epithelial transdifferentiation.
\end{abstract}

\section{Claudin-18 expression under hypoxia in neonatel lungs of brochopulmonary dysplasia model} rats

Jingye Zuo, MM, Yajie Tong, MD, Yuting Yang, MM, Yirui Wang,MM and Dongmei Yue, MD*

Department of Pediatrics, Shengjing Hospital of China Medical University, Shenyang 110004, China

*Corresponding author : Dongmei Yue;Tel:18940255549; Fax number: 024-31939077;yuedm@sjhospital.org

Funding: This research was supported by the Department of Science and Technology of Liaoning Province (grant number $2020 \mathrm{JH} 2 / 10300128$ ) and Natural Science Foundation of Liaoning Province (grant number201602873).

Keywords: Bronchopulmonary dysplasia, Claudin-18, canonical Wnt pathway, alveolar epithelial cell transdifferentiation.

Running head: Claudin-18 in neonatal lungs of BPD model rats

\section{Abstract}

Background: Bronchopulmonary dysplasia (BPD) is characterized by impaired alveolar and microvascular development. Claudin-18 is the only known lung-specific tight junction protein affecting alveolar epithelium development and transdifferentiation. 
Objective: To explore the changes in claudin-18 expression, alveolar epithelial cell (AEC) marker proteins, the canonical Wnt pathway, and their possible regulatory relationships in a hyperoxia-induced BPD rat model.

Methods: The BPD neonatal rat model was established by exposure to hyperoxia (85\%). Hematoxylin and eosin (HE) staining was used to confirm the establishment of the BPD model. The mRNA levels were assessed using quantitative real-time polymerase chain reaction, while protein expression levels were determined using western blotting, immunohistochemical staining, and immunofluorescence .

Results: As confirmed by HE staining, the BPD neonatal rat model was successfully established. Compared with the air group, claudin-18 and claudin-4 expression decreased in the hyperoxia group. The expression of $\beta$-catenin of the Wnt signaling decreased, whereas that of p-GSK-3 $\beta$ increased. Expression of the AEC II marker SFTPC decreased initially and then increased, whereas that of the AEC I marker Podoplanin increased on day $14(\mathrm{P}<0.05)$.

Conclusions: Claudin-18 downregulation during hyperoxia may affect lung development and maturation, which may result in hyperoxia-induced BPD. Additionally, claudin-18 is associated with the canonical Wnt pathway and alveolar epithelial transdifferentiation.

\section{Introduction}

Bronchopulmonary dysplasia (BPD) is one of the most common chronic respiratory diseases in preterm infants. Lung injury that is caused by inflammation is the key cause of BPD (Kalikkot Thekkeveedu et al. 2017; Papagianis et al. 2019), along with impaired alveolar and capillary development that is a typical pathological feature, and pulmonary edema is the main manifestation in the early stage (Bhandari and Bhandari 2003). The alveolar epithelial barrier is a sealed interface between adjacent epithelial cells, and its function depends on tight junctions - a type of transmembrane protein complex.

The claudin family of membrane proteins plays a pivotal role in maintaining the structure and function of tight junctions (Xu et al. 2021). In humans, claudin-1, 3-5, 7, 8, 10, 12, 18, and 23 are most abundantly expressed (Schlingmann et al. 2015). Studies in rats demonstrate that alveolar epithelial cells (AECs) I and II primarily express claudin-3, 4, and 18 (La Femina et al. 2010). However, only claudin-18.1, one of the isomers of claudin-18, is specifically expressed in lung tissues (Türeci et al. 2011), indicating its lung-specific functions. Although claudin-1, 5, 7, 12, 15, and 23 are expressed at low levels in AEC I and II, and may also contribute to the alveolar epithelial barrier function (Tanaka et al. 2017).

In this study, we aimed to assess the changes in claudin-18, claudin-4, SFTPC, Podoplanin, GSK-3 $\beta$, p-GSK$3 \beta$, and $\beta$-catenin expression levels in the lungs of neonatal hyperoxia-induced BDP model rats compared with control rats. Additionally, we aimed to observe the development of alveoli, to further illustrate the role of claudin-18 in the pathophysiological process of hyperoxia-induced BPD.

\section{Materials and Methods}

\subsection{Experimental subjects and group}

All animal experiments were performed in accordance with the National Institutes of Health guidelines and protocols approved by the Institutional Animal Care and Use Committee (approval no. 2020PS574K). Forty Sprague-Dawley rats (10-week-old; Changsheng Co., Ltd. Shenyang, China) were mated at a male to female ratio of 4:1. Female rats were separated after conception. Within $12 \mathrm{~h}$ of the pregnant female rats naturally giving birth, neonatal rats were randomly divided into two groups: air $\left(\left(21 \% \mathrm{O}_{2}, n=50\right)\right.$ and hyperoxia $\left(\left(80-85 \% \mathrm{O}_{2}, n=50\right)\right.$. And the newborn rats with their mother rats were exposed to normoxia or hyperoxia until postnatal day 14. And to avoid oxygen poisoning, the lactating mother rats were switched between normoxia and hyperoxia every $24 \mathrm{~h}$. All animals had a cycle of 12-hour light/dark, free drinking water and standard diet(Vyas-Read et al. 2018). Inhaling isoflurane (2\%) was used for euthanasia.

\subsection{Tissue harvest}


Ten neonatal rats were randomly selected at postnatal days 1, 3, 7, 10, and 14 (Fig. 1) for harvesting their lungs. The thoracic cavity was opened after intraperitoneal anesthesia with $50 \mathrm{~g} / \mathrm{L}$ pentobarbital sodium, and the right ventricle was perfused. When lungs becoming white, they were collected aseptically. For preparing paraffin specimens for HE staining, immunohistochemistry (IHC), and immunofluorescence (IF), the right lower part of the lung was fixed in $4 \%$ paraformaldehyde without dissection, dehydrated using graded alcohol, and rendered transparent using xylene. The remaining lobes were quick-frozen in liquid nitrogen and stored at $-80{ }^{\circ} \mathrm{C}$ for subsequent Western blot analysis and RT-PCR.

\subsection{Determination of wet and dry weight}

To determine the wet and dry weight, the upper right lobe was collected and weighed to determine the wet and dry weight. The lobe was then placed in an oven at $80{ }^{\circ} \mathrm{C}$ for $24 \mathrm{~h}$, upon which it was weighed again; this step was repeated until the weight of the lung lobe no longer changed to get the dry weight. The psychrometric ratio was calculated to assess the degree of pulmonary edema.

\subsection{Hematoxylin and eosin (HE) stanning and radial alveolar count (RAC)}

Paraffin blocks of lung tissues were sliced into 2.5- $\mu \mathrm{m}$ thick sections for HE staining. The radial alveolar count (RAC; Wang et al. 2019) is determined as follows: based on the HE-stained specimens, a straight line is drawn directly between the center of the respiratory bronchioles and the nearest fibrous septum, and the number of alveoli through which the line passes are counted. The RAC was determined using a light microscope $(100 \times$ magnification) by counting each slice thrice and averaging the obtained values.

\subsection{Quantitative real-time PCR}

Total RNA was extracted from lung tissues using the RNAiso Plus (Code No9108, Takara Bio, Japan), and the concentration and purity of the isolated RNA were measured using a NanoDrop spectrophotometer (Thermo Fisher Scientific, MA, USA). For removing genomic DNA contamination and reverse-transcribing the obtained RNA, the PrimeScriptTM RT reagent kit with gDNA Eraser kit (Code No. RRo47A, Takara Bio, Japan) was used. PCR amplification was conducted using qPCR premix (Code No. RR820A, Takara Bio, Japan).Primers were designed by Sangon Biotech; the sequences and other details are shown as follows (Supplementary Table 1).Rat ACTB Endogenous Reference Genes Primers (Order NO. B661202, Sangon Biotech)

The threshold $(\mathrm{Ct})$ value was considered acceptable for analysis. ACTB was used as the reference gene, and the 2- $\Delta \Delta$ Ct method was used to evaluate the relative changes in the expression of each gene. All data shown represent the average results from three replicates.

\subsection{Western blot analysis}

Total protein was extracted from the lung tissue, and its concentration was determined using an enhanced BCA kit (Beyotime Biotechnology). Then, $30 \mu \mathrm{g}$ of the protein sample was subjected to $10 \%$ SDSpolyacrylamide gel electrophoresis, followed by which the separated proteins were transferred to a polyvinylidene fluoride membrane (ISEQ00010, Millipore, USA) (Hu et al. 2021). After blocking with 5\% skim milk at 37 for $2 \mathrm{~h}$, the membrane was incubated with anti-claudin-18 (1:1000, Abcam, USA), anti-claudin-4 (1:1000, Invitrogen, USA), anti-SFTPC (1:1000, Proteintech, China), anti-Podoplanin (1:1000, Santa Cruz, USA), anti-GSK-3 $\beta$ (1:1000, CST,USA), anti-p-GSK-3 $\beta$ (1:1000, CST, USA), and anti- $\beta$-catenin (1: 1000, CST, USA) antibodies at $4{ }^{\circ} \mathrm{C}$ overnight. The next day, the membranes were incubated with the corresponding horseradish peroxide-conjugated secondary antibody (S0001, S0002, Affinity) at room temperature for $2 \mathrm{~h}$. Amersham Imager 600 (GE Healthcare Life Sciences) was used to detect chemiluminescence signals, and the Image $\mathrm{J}$ 1.8.0 was used to calculate the gray value. The experiment was repeated at least thrice.

\subsection{Immunohistochemical staining}

Paraffin sections were deparaffinized, incubated with the Tris-EDTA antigen retrieval solution, and blocked with anti-claudin-18 (1:200), anti-claudin-4 (1:100,), anti-SFTPC (1:100), anti-podoplanin (1:200), and anti- 
$\beta$-catenin (1:100) antibodies at $4{ }^{\circ} \mathrm{C}$ overnight. Images were acquired and analyzed using ImagePro Plus 6.0 to assess protein expression.

\subsection{Immunofluorescence staining}

Paraffin sections (2.5- $\mu$ m thick) were deparaffinized, washed with PBS, heat-repaired, and blocked with 3temperature for $1 \mathrm{~h}$. The specimens were then incubated with anti-claudin-18 (1:200), anti- $\beta$-catenin (1:100), or anti-podoplanin-antibodies $(1: 100)$ overnight at $4{ }^{\circ} \mathrm{C}$. The next day, sections were incubated with Alexa Fluor 488- and Alexa Fluor 594-conjugated secondary antibodies (1:400, Abcam) for $4 \mathrm{~h}$ at room temperature. Sections were subjected to DAPI staining for $5 \mathrm{~min}$, followed by which they were mounted with glycerol and visualized under a full spectrum laser confocal microscope (C1Si, Nikon, Japan).

\subsection{Statistical Analysis}

The data obtained in the experiment are all analyzed using Prism 8.0, and the independent sample T test is used for the comparison of each pair. The statistical significance was set at 0.05 .

\section{Results}

\subsection{Pulmonary edema and alveolar dysfunction observed in the neonatal hyperoxia-induced BPD rat model}

The lung tissues of neonatal rats in the hyperoxia group showed evident edema and immature alveolar development. The lung volume in the hyperoxia group was significantly larger than in the air group $(\mathrm{P}<$ 0.05). The lung tissue was pale, with signs of edema and superficial bleeding spots from days 7 to 10 and slight edema with bleeding spots on day 14. By contrast, the lungs in the air group were uniformly pink with no edema and bleeding spots (Fig. 2A). Psychrometric ratio analysis suggested that the degree of lung tissue edema of neonatal rats in the hyperoxia group was significantly higher than that in the air group (Fig. 2B). Histopathologically, with an increase in age, the alveolar cavity of the air group rats increased in number but decreased in size, and the alveolar compartments increased in number but gradually became thinner. While in the hyperoxia group, the alveolar development gradually became disordered, and the volume of the alveolar cavity became larger with evident alveolar fusion, interstitial cell proliferation, and simultaneous inflammatory cell infiltration (Fig. 2C, 2D).

\subsection{Downregulated claudin-18 and claudin-4 in the hyperoxia-induced BPD rat model}

To confirm the hypothesis, we used different methods to detect the expression of claudin-18 and claudin4 in neonatal lung tissues (Fig. 2A-2I). The qPCR results showed that the claudin-18 transcription of the neonatal lung in the air group was significantly higher than that in the hyperoxia group $(\mathrm{P}<0.05)$ (Fig. 2A). Western blot experiments showed that compared with the air group, claudin-18 expression was significantly reduced to varying degrees under hyperoxia $(\mathrm{P}<0.05)$ (Fig. 2B, 2C). The IHC showed that claudin-18 was mainly expressed in the cytoplasm of AECs (Fig. 2D). The semi-quantitative analysis showed that claudin-18 expression in lung tissues of the air group was significantly higher than that of the oxygen group $(\mathrm{P}<0.05)$ (Fig. 2E). The experimental results are perfectly uniform, seemingly proving that hyperoxia would affect claudin-18 expression in pulmonary tissues.

Similarly, we also used the three methods above to detect another important member of the claudin family, claudin-4. QPCR results showed that claudin-4 transcription in the air group was significantly higher than that in the hyperoxia group on days 10 and 14, whereas no difference in claudin- 4 expression was noted between the air and hyperoxia groups on postnatal days 1,3 , and 7 ( $\mathrm{P}>0.05)$ (Fig. 2F). Western blotting results showed no statistical difference between claudin- 4 expression in the hyperoxia and air groups on postnatal days 1 and $7(\mathrm{P}>0.05)$. However, claudin-4 expression in the air group was significantly higher than that in the hyperoxia group on postnatal days 3, 10, and $14(\mathrm{P}<0.05)$ (Fig. 2B). IHC showed that claudin-4 was primarily expressed in the cytoplasm of AECs and that claudin-4 expression in the hyperoxia group was significantly lower than that in the air group $(\mathrm{P}<0.05)$ (Fig. 2H, 2I). The expression of Claudin-18 and Claudin-4 was decreased in the hyperoxia-induced BPD rat model. 


\subsection{AEC I and AEC II expression in a hyperoxia-induced BPD rat model}

We tested the marker proteins of AEC I-podoplanin and AEC II-SFTPC to confirm the transdifferentiation of AECs under hyperoxia (Fig. 4A-4I). The RT-PCR results showed that except for day 1, Podoplanin in the hyperoxia group was significantly higher than that in the air group (Fig. 4A). Western blot results showed that claudin-18 expression only increased significantly on postnatal day 14, with no difference between the expression levels in the hyperoxia and air groups on days 1, 3, 7, and 10 (Fig. 3B, 3C). Similar to the RTPCR results, IHC showed that under hyperoxic conditions, Podoplanin expression was significantly increased (Fig. 3D, 3E).

Unlike podoplanin, the AEC II marker SFTPC showed good consistency. The SFTPC transcription of neonatal lungs in the hyperoxia group was significantly lower than that in the air group on postnatal days 1, 3, 7, and 10 but significantly higher on day 14 (Fig. 4F). Western blot results suggested that the SFTPC in the hyperoxia group was significantly lower than that in the air group on postnatal days 3 and 7 , whereas its expression was statistically higher in the hyperoxia group on postnatal days 10 and 14 (Fig. 4B, 4G). IHC suggested that SFTPC was expressed in the nucleus and cytoplasm (Fig. 4H). The semi-quantitative analysis results were consistent with the Western blot results (Fig. 4I).

\subsection{Wnt canonical pathway was inhibited in the hyperoxia-induced BPD rat model}

After experimental verification, the Wnt pathway was downregulated under hyperoxia (Fig. 5A-5F). The mRNA expression of $\beta$-catenin in the hyperoxia group was also significantly lower than that in the air group (Fig. 5A). Western blot results showed no statistical difference in the $\beta$-catenin expression between the two groups on postnatal day 3 ; the expression in the hyperoxia group was significantly lower than that in the air group on postnatal days $1,7,10$, and 14 ; the $\beta$-catenin level was dramatically reduced on day 14 (Fig. 6B, $6 \mathrm{C}$ ). In addition, the relative protein expression of p-GSK-3 $\beta$ significantly increased on days 7 and 10 (Fig. $5 \mathrm{D}$ ). IHC showed that $\beta$-catenin was expressed in the nucleus and cytoplasm (Fig. 5E). Under hyperoxia conditions, $\beta$-catenin expression was significantly reduced (Fig. $5 \mathrm{~F}$ ), mainly in the cytoplasm. These results were confirmed by IHC.

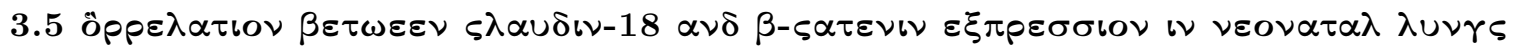

Double IF staining was performed to localize claudin-18 and podoplanin in pulmonary tissues. Claudin-18 was mainly expressed in the cytoplasm around the cell membrane, while podoplanin was mainly expressed in the cytoplasm. Overall, at the same time point, compared with the oxygen group, the green fluorescence intensity of claudin-18 was higher in the air group; the red fluorescence intensity of podoplanin was lower, so the color was greener, whereas the fluorescence color of the oxygen group was reddish (Fig. 6A).

To observe the effect of hyperoxia on claudin-18 and $\beta$-catenin expression, we performed double IF staining of claudin-18 and $\beta$-catenin (Fig. 6B). As before, claudin-18 was mainly expressed at the edge of the cytoplasmic cell membrane, and $\beta$-catenin was diffusely expressed in the cytoplasm. The fluorescence intensity of both was greatly reduced under hyperoxic conditions. The red represented by $\beta$-catenin underwent a greater reduction, and the fluorescence of the air group was therefore orange, whereas the color of the oxygen group was greener.We conducted a Pearson correlation analysis on claudin-18 and $\beta$-catenin expression levels; the results demonstrated a significant positive correlation between claudin- 18 and $\beta$-catenin protein expression $(\mathrm{r}=0.286, \mathrm{P}<0.05)($ Fig. $6 \mathrm{C})$.

\section{Discussion}

In this study, we aimed to determine the role of claudin-18 in the pathophysiology of hyperoxia-induced BPD using a neonatal rat model of hyperoxia-induced BPD. Claudin-18 was mainly distributed at the edge of the cell membrane in the cytoplasm. Compared with the air group, there was a significant decrease in claudin-18 expression under hyperoxic conditions. SFTPC expression was reduced at postnatal days 3 and 7 , while an increase in expression was noted at postnatal days 10 and 14. Podoplanin expression was increased on postnatal day 14. Our preliminary study revealed that the transdifferentiation of AECs was interrupted 
under hyperoxia. Overall, our results showed that the classic Wnt/ $\beta$-catenin pathway was inhibited under hyperoxia.

The spatiotemporal expression pattern of claudin-18 during lung development is highly regulated. From embryo day 16.5 to postnatal day 15, its expression shows an upward trend. Except for postnatal day 3, claudin-18 expression declines then reaches a peak at postnatal day 15 (Lewis et al. 2018). In various animal models of lung injury, decreased claudin-18 expression has been considered a sign of lung barrier damage (Lee et al. 2020; M et al. 2020; Reynolds et al. 2018; Weber et al. 2019). Especially in a hyperoxia-induced BPD model, claudin-18 may be involved in early pulmonary edema development and late alveolar development (Abdul-Hafez et al. 2019).

La Femina et al. (2014) studied the role of claudin-18 in lung development and barrier function in a claudin-18 (exon 2 and 3) knock-out (KO) mouse model. They found that, compared with wild-type mice, claudin$18 \mathrm{KO}$ mice showed lung injury and pulmonary edema, whose severity increased with time, along with an increased alveolar epithelial barrier permeability. When AECs are formed in claudin-18 KO mice, an increase in radial perinuclear aggregates associated with the nuclear plasma membrane is reportedly observed (Jin et al. 2013). The changes in the morphology and function of tight junctions noted in the claudin- $18 \mathrm{KO}$ mouse model were alike those observed in the rat model of hyperoxia-induced BPD, namely fewer alveoli, larger in size, widened alveolar compartments, and impaired alveolar formation. Human fetal alveoli with a gestational age of 23-24 weeks, which are in the small vesicle stage and still immature, show a state of atelectasis, widened alveolar interval and increased alveolar fluid exudation. These changes occur within the risk period for BPD development (Day and Ryan 2017) and may be related to abnormal claudin expression.

Our research showed increased SFTPC expression in neonatal lungs under hyperoxia. Although the transdifferentiation from AEC II to AEC I was thought to be enhanced under these conditions, SFTPC expression was observed to decrease at the early stage and increase later, contrasting the expected trend of transdifferentiation enhancement. These results are consistent with those obtained in previous studies. Hou et al. (2015) found that SFTPC expression in tissues first decreased and then increased, while that of the AEC I marker, AQP5, increased in the BPD model. However, primary cells isolated from lung tissues indicated decreasing SFTPC expression and increasing AQP5 expression. The reason may be that when AEC II transdifferentiation to AEC I increases in tissues, AEC II proliferation is required to maintain the number of cells. In earlier studies, Chen et al. (2007) explored the differences in the expression of genes related to AEC II proliferation and differentiation. They found that the expression of SPC and SPA was decreased, while that of T1a (an AEC I marker) was decreased, supporting the notion of transdifferentiation. Another paper investigating the contents of SP-C and SP-D in bronchoalveolar lavage fluid by enzyme-linked immunosorbent assay suggested that the SPC content was higher in the hyperoxia group on day 3 , reached its peak on day 7, and then began to decrease on days 10 and 14 (Jin et al. 2018). The increase in SFTPC expression might also be caused by the failure to release SFTPC into the alveolar cavity due to pulmonary edema.

Furthermore, some scholars found that the expression of claudin-18 and alveolar surfactants (SPA, SPB, and SPC) in MLE-12 cells was significantly increased following corticosteroid analogs treatment. Silencing claudin-18 effectively inhibited corticosteroid analog-induced SPB and SPC expression (Shi et al. 2019). Therefore, claudin-18 expression was deemed related to peripheral lung epithelial cell maturation. Furthermore, drugs that promote lung maturation would induce claudin-18 expression in human fetal AECs and promote alveolar development, suggesting that claudin-18 may be a potential driver of lung maturation (Niimi et al. 2001). In addition, compared with the wild-type group, higher expression of pro-SPC and Podoplanin was observed in the claudin-18 KO mouse model (La Femina et al. 2014). The researchers speculated that the KO of claudin-18 could have resulted in abnormal AEC differentiation. However, the extent to which claudin-18 expression affects alveolar epithelial cell phenotype needs further research, and the mechanisms underlying barrier dysfunction and AEC damage remain unclear.

In our study, the Wnt signaling pathway was inhibited under hyperoxia, possibly enhancing AEC II transdifferentiation. However, the Wnt signaling pathway is reactivated during alveolar formation, leading to AEC II proliferation. In contrast, inhibition of this pathway could reduce cell proliferation, promoting AEC II 
differentiation to AEC I (Frank et al. 2016). The Wnt signaling pathway is crucial in embryonic development and is significantly involved in lung diseases (Ota et al. 2016). The expression of $\beta$-catenin in embryonic lung respiratory epithelial cells is necessary for the growth and differentiation of peripheral epithelial cell progenitor cells (Mathew 2020). The absence of $\beta$-catenin in embryonic lung epithelial cells disrupts lung morphogenesis, restricts the formation and differentiation of peripheral lungs, and enhances the formation of conductive airways (Mucenski et al. 2003).

However, many recent studies have reported that the Wnt signaling pathway is activated by hyperoxia (Alapati et al. 2011; Alapati et al. 2013; Xu et al. 2015). Our results differ from these previously reported results in at least two aspects. First, we tested five time points after birth, a critical period for lung development, when the normal development of the lung is ensured by the precise regulation of the Wnt pathway. In addition, experiments yielding opposite results were conducted under the intervention of different drugs; therefore, we cannot rule out that the placebo used did not affect the expression of the Wnt pathway. Furthermore, even though the BPD model was established similarly to previous studies, the feeding conditions and animal batches used differed. Hence, results are expectedly inconsistent with those of some previous studies.

We found that, in the hyperoxia-induced BPD model, claudin-18 and $\beta$-catenin expression simultaneously decreased (Fig. 8). We speculated that downregulating claudin 18 expression inhibits the classic Wnt signaling pathway (Lingappan and Savani 2020). However, the evidence obtained in this study is insufficient to confirm this speculation. In the future, we need to clarify the regulatory effect of claudin-18 on the Wnt/ $\beta$-catenin signaling pathway and determine the process underlying claudin-18-mediated inhibition of the Wnt/ $\beta$-catenin signaling pathway under hyperoxic conditions.

This is the first study to use a neonatal rat model of hyperoxia-induced BPD to study the effect of claudin-18 on rat alveolar epithelial transdifferentiation. However, a limitation of our study is that we only conducted research through animal experiments and did not explore the molecular mechanisms underlying the obtained results.

\section{Conclusions}

In conclusion, in a neonatal rat model of hyperoxia-induced BPD used, changes in claudin-18 and claudin- 4 expression were found to follow the same trend as that of the classic Wnt signaling pathway, co-occurring with abnormal AEC transdifferentiation. Therefore, the claudin protein may be a potential regulator of the Wnt canonical pathway and may play a unique role in AEC transdifferentiation. Nonetheless, this hypothesis warrants further experimental validation.

\section{Acknowledgments}

This research was partially supported by the Department of Science and Technology of Liaoning Province (grant number $2020 \mathrm{JH2} / 1030$ ) and the Natural Science Foundation of Liaoning Province (grant number201602873).

\section{Conflict of Interest}

The authors declare that there is no conflict of interest regarding the publication of this paper.

\section{References}

Abdul-Hafez A, Mohamed T, Uhal BD. 2019. Activation of mas restores hyperoxia-induced loss of lung epithelial barrier function through inhibition of apoptosis. J Lung Pulm Respir Res. 6(3):58-62.

Alapati D, Rong M, Chen S, Hehre D, Rodriguez MM, Lipson KE, Wu S. 2011. Connective tissue growth factor antibody therapy attenuates hyperoxia-induced lung injury in neonatal rats. Am J Respir Cell Mol Biol. 45(6):1169-1177.

Alapati D, Rong M, Chen S, Lin C, Li Y, Wu S. 2013. Inhibition of lrp5/6-mediated wnt/ $\beta$-catenin signaling by mesd attenuates hyperoxia-induced pulmonary hypertension in neonatal rats. Pediatr Res. 73(6):719-725. 
Bhandari A, Bhandari V. 2003. Pathogenesis, pathology and pathophysiology of pulmonary sequelae of bronchopulmonary dysplasia in premature infants. Front Biosci. 8:e370-380.

Chen Z, Chintagari NR, Guo Y, Bhaskaran M, Chen J, Gao L, Jin N, Weng T, Liu L. 2007. Gene expression of rat alveolar type ii cells during hyperoxia exposure and early recovery. Free Radic Biol Med. 43(4):628-642.

Day CL, Ryan RM. 2017. Bronchopulmonary dysplasia: New becomes old again! Pediatr Res. 81(1-2):210213.

Frank DB, Peng T, Zepp JA, Snitow M, Vincent TL, Penkala IJ, Cui Z, Herriges MJ, Morley MP, Zhou S et al. 2016. Emergence of a wave of wnt signaling that regulates lung alveologenesis by controlling epithelial self-renewal and differentiation. Cell Rep. 17(9):2312-2325.

Hou A, Fu J, Yang H, Zhu Y, Pan Y, Xu S, Xue X. 2015. Hyperoxia stimulates the transdifferentiation of type ii alveolar epithelial cells in newborn rats. Am J Physiol Lung Cell Mol Physiol. 308(9):L861-872.

Hu J, Wang J, Li C, Shang Y. 2021. Fructose-1,6-bisphosphatase aggravates oxidative stress-induced apoptosis in asthma by suppressing the nrf2 pathway. J Cell Mol Med. 25(11):5001-5014.

Jin W, Rong L, Liu Y, Song Y, Li Y, Pan J. 2013. Increased claudin-3, -4 and -18 levels in bronchoalveolar lavage fluid reflect severity of acute lung injury. Respirology. 18(4):643-651.

Jin Y, Peng LQ, Zhao AL. 2018. Hyperoxia induces the apoptosis of alveolar epithelial cells and changes of pulmonary surfactant proteins. Eur Rev Med Pharmacol Sci. 22(2):492-497.

Kalikkot Thekkeveedu R, Guaman MC, Shivanna B. 2017. Bronchopulmonary dysplasia: A review of pathogenesis and pathophysiology. Respir Med. 132:170-177.

LaFemina MJ, Rokkam D, Chandrasena A, Pan J, Bajaj A, Johnson M, Frank JA. 2010. Keratinocyte growth factor enhances barrier function without altering claudin expression in primary alveolar epithelial cells. Am J Physiol Lung Cell Mol Physiol. 299(6):L724-734.

LaFemina MJ, Sutherland KM, Bentley T, Gonzales LW, Allen L, Chapin CJ, Rokkam D, Sweerus KA, Dobbs LG, Ballard PL et al. 2014. Claudin-18 deficiency results in alveolar barrier dysfunction and impaired alveologenesis in mice. Am J Respir Cell Mol Biol. 51(4):550-558.

Lee PH, Hong J, Jang AS. 2020. N-acetylcysteine decreases airway inflammation and responsiveness in asthma by modulating claudin 18 expression. Korean J Intern Med. 35(5):1229-1237.

Lewis JB, Jimenez FR, Merrell BJ, Kimbler B, Arroyo JA, Reynolds PR. 2018. The expression profile of claudin family members in the developing mouse lung and expression alterations resulting from exposure to secondhand smoke (shs). Exp Lung Res. 44(1):13-24.

Lingappan K, Savani RC. 2020. The wnt signaling pathway and the development of bronchopulmonary dysplasia. Am J Respir Crit Care Med. 201(10):1174-1176.

M T, T A, B S, Ak G, Sks S. 2020. Curcumin prophylaxis refurbishes alveolar epithelial barrier integrity and alveolar fluid clearance under hypoxia. Respir Physiol Neurobiol. 274:103336.

Mathew R. 2020. Signaling pathways involved in the development of bronchopulmonary dysplasia and pulmonary hypertension. Children (Basel). 7(8).

Mucenski ML, Wert SE, Nation JM, Loudy DE, Huelsken J, Birchmeier W, Morrisey EE, Whitsett JA. 2003. Beta-catenin is required for specification of proximal/distal cell fate during lung morphogenesis. J Biol Chem. 278(41):40231-40238.

Niimi T, Nagashima K, Ward JM, Minoo P, Zimonjic DB, Popescu NC, Kimura S. 2001. Claudin-18, a novel downstream target gene for the t/ebp/nkx2.1 homeodomain transcription factor, encodes lung- and stomach-specific isoforms through alternative splicing. Mol Cell Biol. 21(21):7380-7390. 
Ota C, Baarsma HA, Wagner DE, Hilgendorff A, Königshoff M. 2016. Linking bronchopulmonary dysplasia to adult chronic lung diseases: Role of wnt signaling. Mol Cell Pediatr. 3(1):34.

Papagianis PC, Pillow JJ, Moss TJ. 2019. Bronchopulmonary dysplasia: Pathophysiology and potential anti-inflammatory therapies. Paediatr Respir Rev. 30:34-41.

Reynolds CJ, Quigley K, Cheng X, Suresh A, Tahir S, Ahmed-Jushuf F, Nawab K, Choy K, Walker SA, Mathie SA et al. 2018. Lung defense through il-8 carries a cost of chronic lung remodeling and impaired function. Am J Respir Cell Mol Biol. 59(5):557-571.

Schlingmann B, Molina SA, Koval M. 2015. Claudins: Gatekeepers of lung epithelial function. Semin Cell Dev Biol. 42:47-57.

Shi F, Liao Y, Dong Y, Wang Y, Xie Y, Wan H. 2019. Claudin18 associated with corticosteroid-induced expression of surfactant proteins in pulmonary epithelial cells. J Matern Fetal Neonatal Med. 32(5):809-814.

Tanaka H, Tamura A, Suzuki K, Tsukita S. 2017. Site-specific distribution of claudin-based paracellular channels with roles in biological fluid flow and metabolism. Ann N Y Acad Sci. 1405(1):44-52.

Türeci O, Koslowski M, Helftenbein G, Castle J, Rohde C, Dhaene K, Seitz G, Sahin U. 2011. Claudin-18 gene structure, regulation, and expression is evolutionary conserved in mammals. Gene. 481(2):83-92.

Vyas-Read S, Vance RJ, Wang W, Colvocoresses-Dodds J, Brown LA, Koval M. 2018. Hyperoxia induces paracellular leak and alters claudin expression by neonatal alveolar epithelial cells. Pediatr Pulmonol. $53(1): 17-27$.

Wang J, Zhang A, Li Y, Xu J, Huang F, Zhao M, Wu B, He S. 2019. Effect of intermittent hypoxia or hyperoxia on lung development in preterm rat neonates during constant oxygen therapy. J Cell Biochem. 120(10):17545-17554.

Weber B, Mendler MR, Lackner I, von Zelewski A, Hofler S, Baur M, Braun CK, Hummler H, Schwarz S, Pressmar J et al. 2019. Lung injury after asphyxia and hemorrhagic shock in newborn piglets: Analysis of structural and inflammatory changes. PLoS One. 14(7):e0219211.

Xu W, Zhao Y, Zhang B, Xu B, Yang Y, Wang Y, Liu C. 2015. Resveratrol attenuates hyperoxia-induced oxidative stress, inflammation and fibrosis and suppresses wnt/ $\beta$-catenin signalling in lungs of neonatal rats. Clin Exp Pharmacol Physiol. 42(10):1075-1083.

Xu YN, Deng MS, Liu YF, Yao J, Xiao ZY. 2021. Tight junction protein cldn17 serves as a tumor suppressor to reduce the invasion and migration of oral cancer cells by inhibiting epithelial-mesenchymal transition. Arch Oral Biol. 133:105301.

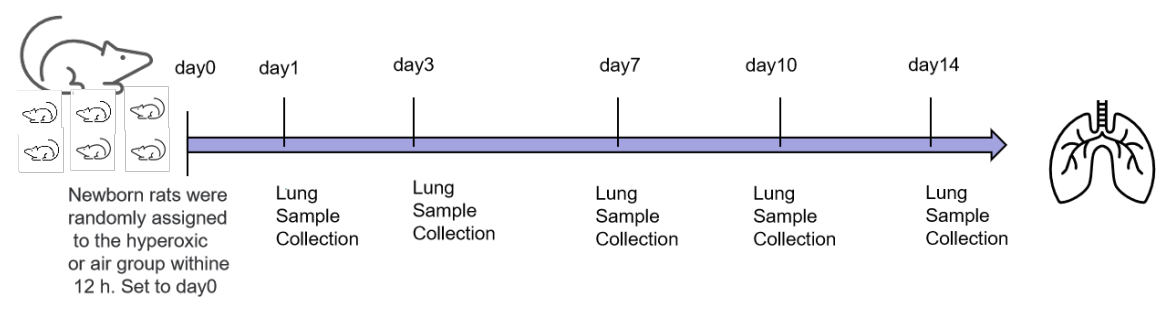



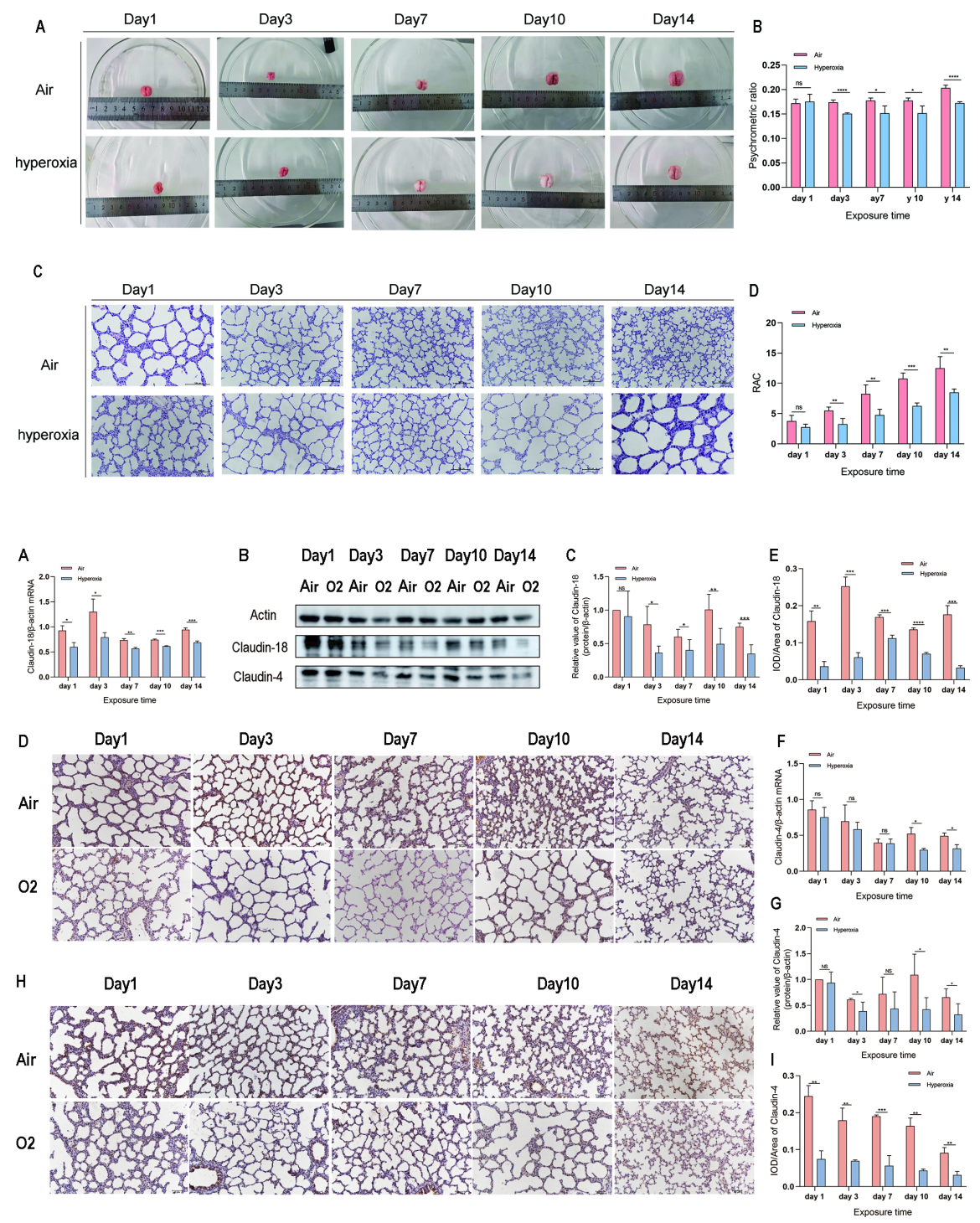


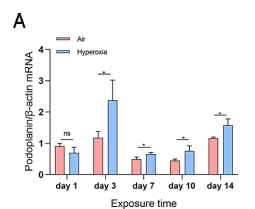

B Day1 Day3 Day7 Day10 Day14 Air 02 Air 02 Air 02 Air 02 Air 02

Actin $-\cdots-\cdots \cdots \cdots$

SFTPC $-\mathbf{3}-\cdots=\ldots$

Podoplanin

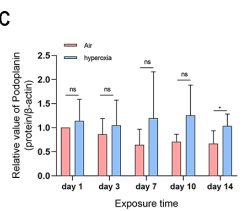

E

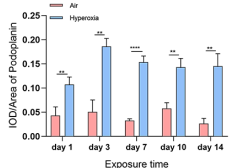

F
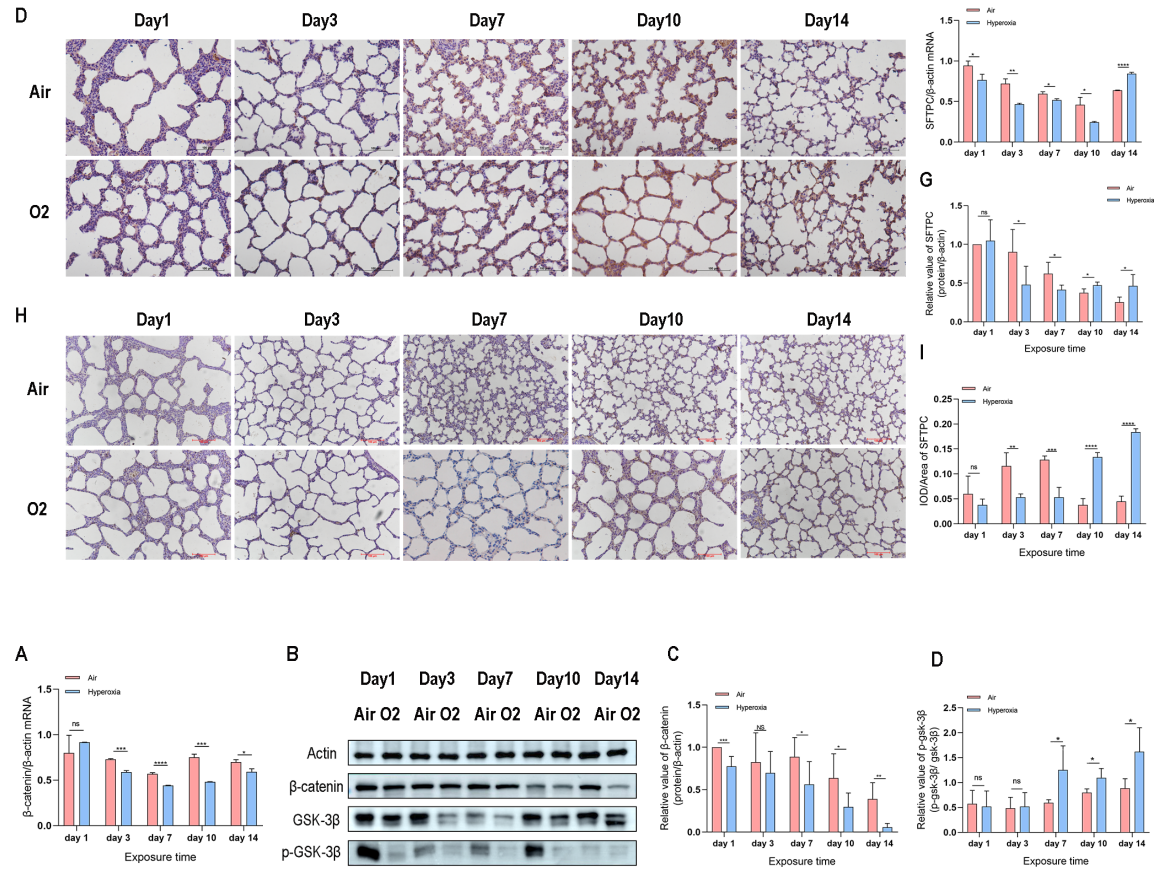

E

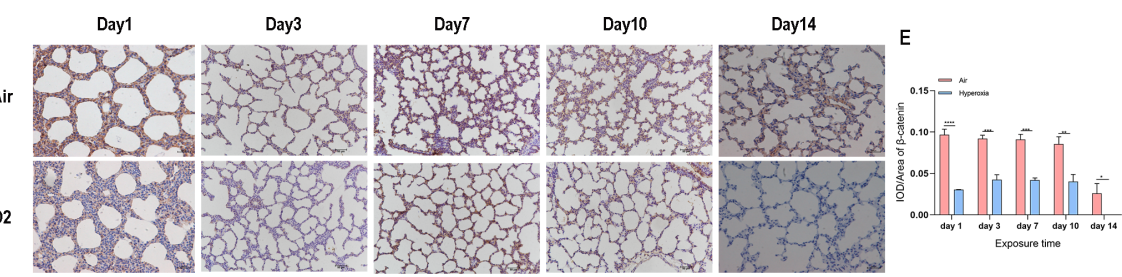


A

Day 3

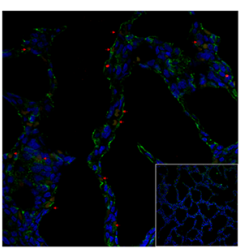

Hyperoxia

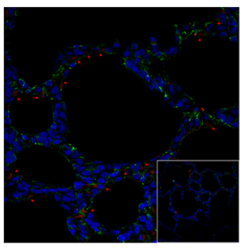

B
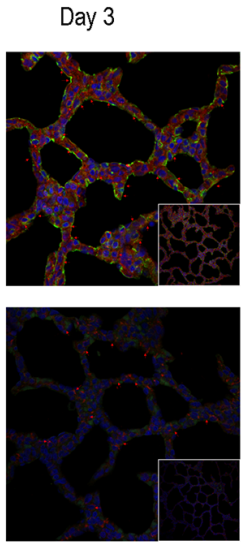

Hyperoxia
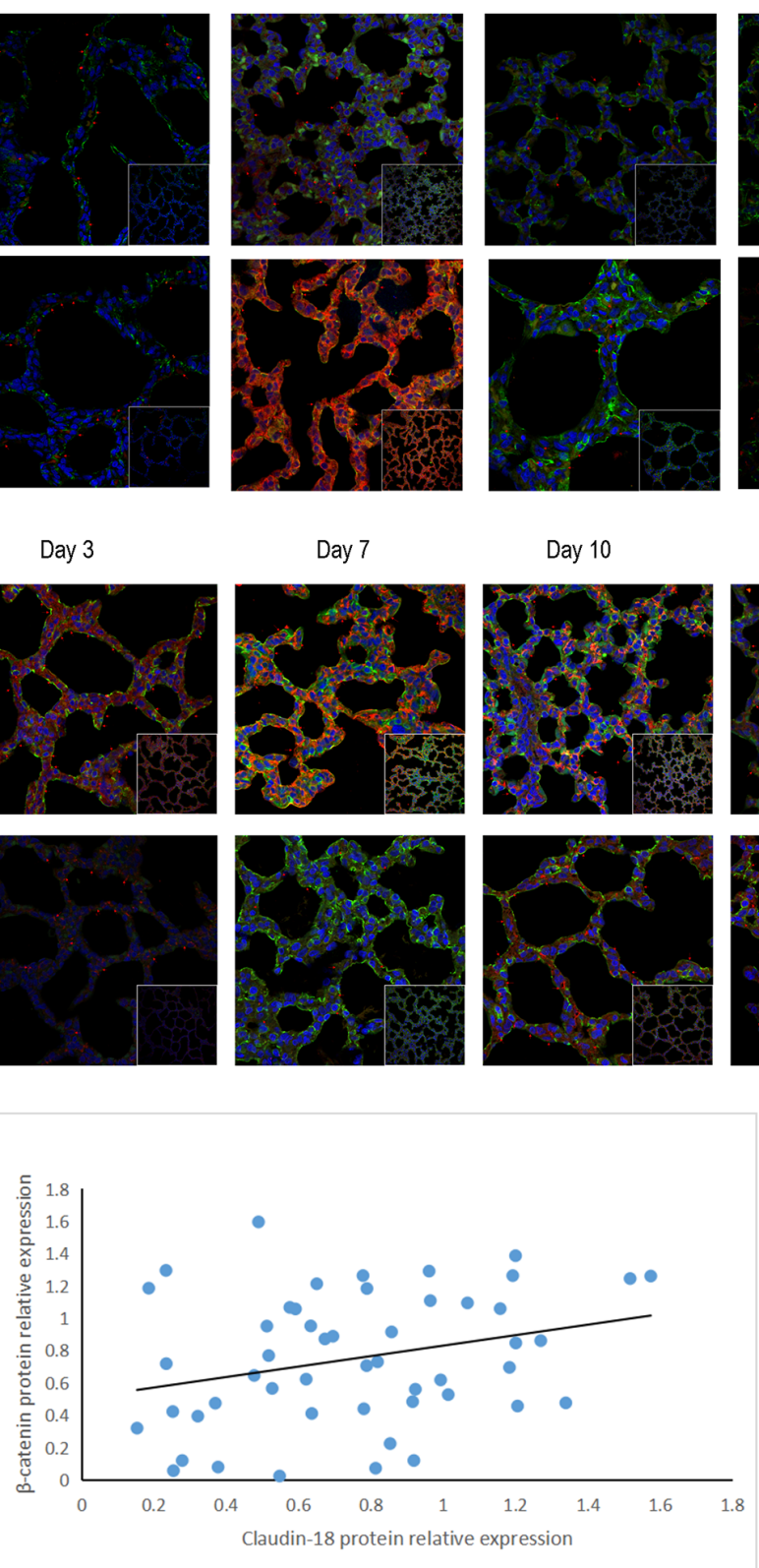

Day 10

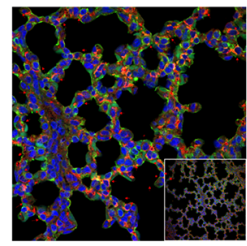

C
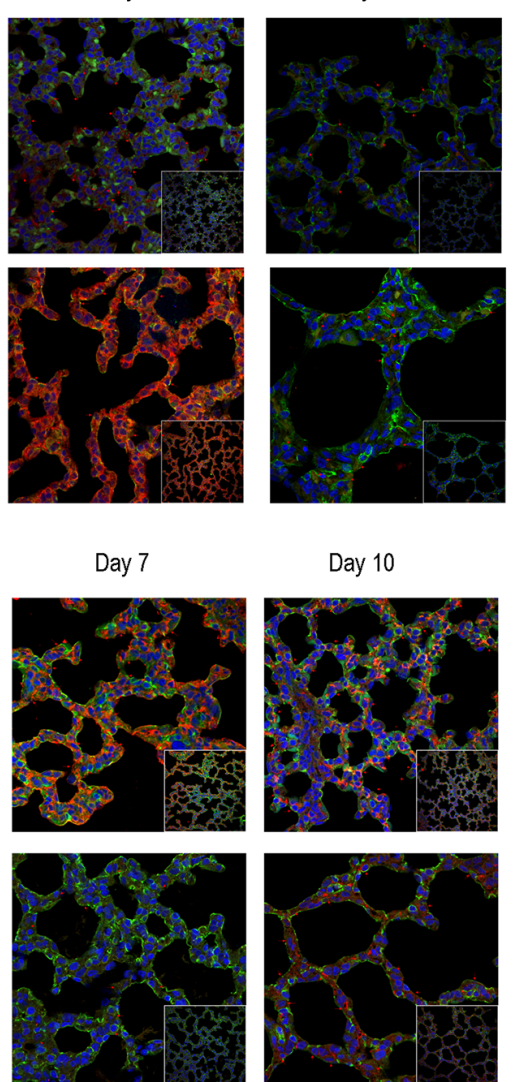

Day 14
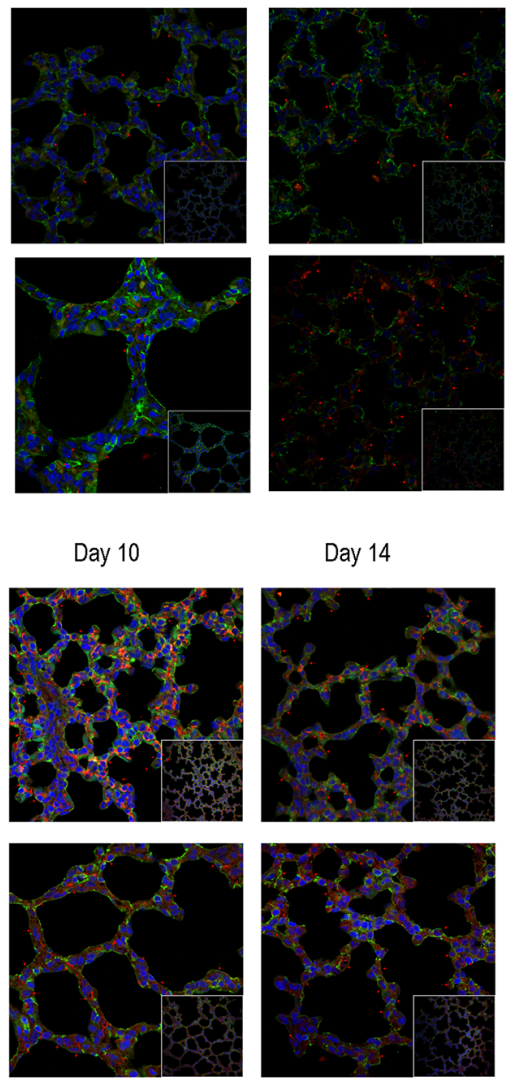

Day 14

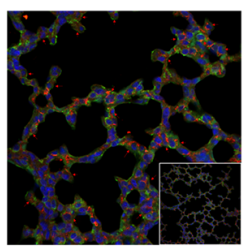

